\title{
Evolutionary Divide and Conquer (I): A Novel Genetic Approach to the TSP
}

\author{
Christine L. Valenzuela \\ Department of Computing \\ Imperial College of Science, \\ Technology and Medicine \\ 180 Queen's Gate \\ London SW7 2BZ \\ United Kingdom
}

\author{
Antonia J. Jones \\ Department of Computing \\ Imperial College of Science, \\ Technology and Medicine \\ 180 Queen's Gate \\ London SW7 2BZ \\ United Kingdom
}

\begin{abstract}
Experiments with genetic algorithms using permutation operators applied to the traveling salesman problem (TSP) tend to suggest that these algorithms fail in two respects when applied to very large problems: they scale rather poorly as the number of cities $n$ increases, and the solution quality degrades rapidly. We propose an alternative approach for genetic algorithms applied to hard combinatoric search which we call Evolutionary Divide and Conquer (EDAC). This method has potential for any search problem in which knowledge of good solutions for subproblems can be exploited to improve the solution of the problem itself. The idea is to use the genetic algorithm to explore the space of problem subdivisions rather than the space of solutions themselves. We give some preliminary results of this Keywords method applied to the geometric TSP.
\end{abstract}

evolutionary algorithms, geometric TSP, divide and conquer, Karp's algorithm.

\section{Introduction}

Our experience with genetic algorithms using permutation operators applied to the geometric traveling salesman problem (TSP) suggests that these algorithms fail in two respects when applied to very large problems: they scale rather poorly as the number of cities $n$ increases, and the solution quality degrades rapidly. We shall present detailed results to illustrate these observations in a more comprehensive discussion. However, our goal here is to describe a new approach we are developing that is designed to overcome these problems.

We call our alternative method, for genetic algorithms applied to hard combinatoric search, Evolutionary Divide and Conquer (EDAC). This approach has potential for any search problem in which knowledge of good solutions for subproblems can be exploited to improve the solution of the problem itself. The idea is to use the genetic algorithm to explore the space of problem subdivisions rather than the space of solutions themselves. We give some preliminary results of this method applied to the geometric TSP. Essentially, we are suggesting that intrinsic parallelism is no substitute for divide and conquer in hard combinatoric search, and we aim to have both.

Our goal has been to develop a genetic algorithm capable of producing reasonable quality solutions for problems of several thousand cities, and one that will scale well as the problem size $n$ increases. "Scaling well" in this context almost inevitably means a time complexity of $O(n)$ or at worst $O(n \log n)$. This is a fairly severe constraint; for example, given a list of 
$n$ city coordinates the simple act of computing all possible edge lengths, a $O\left(n^{2}\right)$ operation, is excluded. Such an operation may be tolerable for $n=5000$ but becomes intolerable for $n=100,000$.

Given the self-imposed scaling constraint, the other two important axes of comparison are the quality of solutions and the actual run time. To provide some basis for comparison we contrast our approach with the standard 2-Opt.

\subsection{TSP Algorithms}

The best exact solution methods for the traveling salesman problem are capable of solving problems of several hundred cities (Grötschel \& Holland, 1991), but unfortunately excessive amounts of computer time are used in the process and, as $n$ increases, any exact solution method rapidly becomes impractical. For large problems we therefore have no way of knowing the exact solution, but in order to gauge the solution quality of any algorithm we need a reasonably accurate estimate of the minimal tour length. This is usually provided in one of two ways.

For a uniform distribution of cities the classic work by Beardwood, Halton, and Hammersley (BHH) (1959) obtains an asymptotic, best-possible upper bound for the minimum tour length for large $n$. Let $\left\{X_{i}\right\}, 1 \leq i<\infty$, be independent random variables uniformly distributed over the unit square, and let $L_{n}$ denote the shortest closed path that connects all the elements of $\left\{X_{1}, \ldots, X_{n}\right\}$. In the case of the unit square they proved, for example, that there is a constant $c>0$ such that, with probability 1 ,

$$
\lim _{n \rightarrow \infty} L_{n} n^{-1 / 2}=c
$$

where $c>0$ is a constant. In general $c$ depends on the geometry of the region considered.

One can use the estimate provided by the $\mathrm{BHH}$ theorem in the following form: the expected length $L_{n}^{*}$ of a minimal tour for an $n$-city problem, in which the cities are uniformly distributed in a square region of the Euclidean plane, is given by

$$
L_{n}^{*} \approx 0.765 \sqrt{n R}
$$

where $R$ is the area of the square and the constant 0.765 has been determined empirically (Stein, 1977). In all our experiments we fix the area $R$ so that $L_{n}^{*}=100$ and the percentage excess of a tour length is the percentage excess relative to this estimate.

A second possibility would be to use a problem-specific estimate of the minimal tour length, which gives a very accurate estimate: the Held-Karp lowver bound (Held \& Karp, 1970, 1971). Computing the Held-Karp lower bound is an iterative process involving the evaluation of Minimal Spanning Trees for $n-1$ cities of the TSP followed by Lagrangean relaxations. However, the typical percentage excess of the present version of our algorithm does not really require us to implement this estimate.

If one seeks approximate solutions then various algorithms based on simple rule-based heuristics (e.g., nearest neighbor and greedy heuristics), or local search tour improvement heuristics (e.g., 2-Opt, 3-Opt, and Lin-Kernighan), can produce good-quality solutions much faster than exact methods. A combinatorial local search algorithm is built around a "combinatoric neighborhood search" procedure, which, given a tour, examines all tours that are closely related to it and finds a shorter "neighboring" tour, if one exists. Algorithms of this type are discussed by Papadimitriou and Steiglitz (1982). The definition of "closely related" varies with the details of the particular local search heuristic.

The particularly successful combinatorial local search heuristic described by Lin and Kernighan (1973) defines "neighbors" of a tour to be those tours that can be obtained from 
it by doing a limited number of interchanges of tour edges with nontour edges. The slickest local heuristic algorithms, ${ }^{1}$ which on average tend to have complexity $O\left(n^{\alpha}\right)$, for $\alpha>2$, can produce solutions with approximately $1-2 \%$ excess for 1000 cities in a few minutes. However, for 10,000 cities the time escalates rapidly, and one might expect that the solution quality also degrades (see Gorges-Schleuter, 1990, p. 101).

An approximation scbeme $\mathrm{A}$ is an algorithm that, given problem instance $I$ and $\varepsilon>0$, returns a solution of length $A(I, \varepsilon)$ such that

$$
\frac{\left|A(I, \varepsilon)-L_{n}(I)\right|}{L_{n}(I)} \leq \varepsilon
$$

Such an approximation scheme is called a fully polynomial time approximation scheme if its run time is bounded by a function that is polynomial in both the instance size and $1 / \varepsilon$. Unfortunately, the following theorem holds (see, for example, Lawler, Lenstra, Rinnooy Kan, and Shmoys, 1985, pp. 165-166).

Theorem. If $\mathcal{P} \neq N \mathcal{P}$ then there can be no fully polynomial time approximation scheme for the $T S P$, even if instances are restricted to points in the plane under the Euclidean metric.

Although the possibility of a fully polynomial time approximation scheme is effectively ruled out, there remains the possibility of an approximation scheme that although not polynomial in $1 / \varepsilon$, does have a running time that is polynomial in $n$ for every fixed $\varepsilon>0$. The Karp algorithms, based on cellular dissection, provide "probabilistic" approximation schemes for the geometric TSP.

Theorem (Karp, 1977). For every $\varepsilon>0$ there is an algoritbm $A(\varepsilon)$ such that $A(\varepsilon)$ runs in time $C(\varepsilon) n+O(n \log n)$ and, with probability $1, A(\varepsilon)$ produces a tour of lengtb not more than $1+\varepsilon$ times the length of a minimal tour.

The Karp-Steele algorithms (Steele, 1986) can in principle converge in probability to near-optimal tours very rapidly. Cellular dissection is a form of divide and conquer. Karp's algorithms partition the region $R$ into small subregions, each containing about $t$ cities. An exact or heuristic method is then applied to each subproblem, and the resulting subtours are finally patched together to yield a tour through all the cities.

To date, the best genetic algorithms designed for TSP problems have used permutation crossovers (Davis, 1985; Goldberg \& Lingle, 1985; Smith, 1985), or edge recombination operators (Whitley, 1989), and required massive computing power to gain very good approximate solutions (often actually optimal) to problems with a few hundred cities (GorgesSchleuter, 1990). Gorges-Schleuter cleverly exploited the architecture of a transputer bank to define a topology on the population and introduce local mating schemes that enabled her to delay the onset of premature convergence. However, this improvement to the genetic algorithm is independent of any limitations inherent in permutation crossovers.

\subsection{Genetic Algorithms Based on Karp's Approach}

In practice, a one-shot deterministic Karp algorithm yields rather poor solutions, typically $30 \%$ excess (with simple patching) when applied to 500-1000 city problems. Nevertheless, we believe it is a good starting point for exploring $E D A C$ applied to the TSP. Our reasons

1 The most impressive results in this direction are due to David Johnson at AT\&T Bell Laboratories-mostly reported in unpublished workshop presentations. 


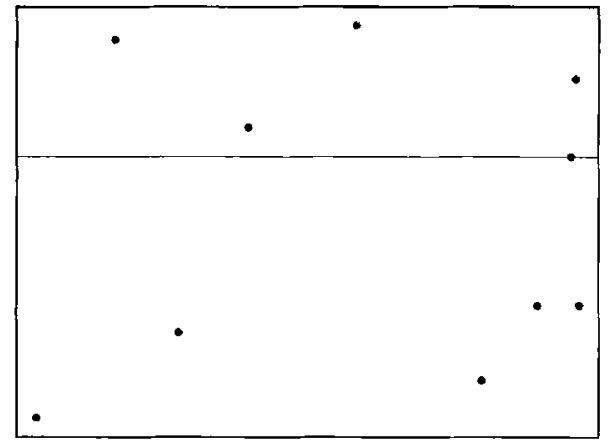

Figure 1. Horizontal bisection of a 10-city problem.

are twofold. First, there is some probabilistic asymptotic guarantee of solution quality as the problem size increases. Second, the time complexity is about as good as one can hope for, namely $O(n \log n)$. The run time of a genetic algorithm based on exploring the space of "Karp-like" solutions will be proportional to $n \log n$ multiplied by the number of times the Karp algorithm is run, i.e., the number of individuals tested.

Thus, we have reasonable probabilistic guarantees for both the complexity and the solution quality. For large enough problems, several thousand Karp runs (individuals tested) will be much faster than a combinatorial local search heuristic algorithm. The practical objection might very well be that "large enough" turns out to be very large indeed, but still this would seem to be an approach worthy of study.

\section{Developing a Divide-and-Conquer Approach}

\subsection{Bisection Method 1}

Let rectangle $R$ contain $m$ cities. Let $y$ be the $y$-coordinate of the $[m / 2] t h$ closest city to the top edge of $R$. A horizontal cut through $y$ subdivides $R$ into two rectangles, an upper rectangle and a lower rectangle. The situation is illustrated in Figure 1. The effect is to place half the cities either side of the bisecting line with at least one city on the bisector. In a similar fashion, a vertical cut could be applied to bisect the cities through $x$, which is the $x$-coordinate of the $[m / 2] t b$ closest city to the left edge of $R$. In Karp's first algorithm, the direction of the cut is always parallel to the shorter side of the rectangle. Karp showed that by minimizing the lengths of the perimeters of the rectangles he was able to minimize the expected lengths of the tours. The preliminary results reported in section 4 used this method of bisection.

\subsection{Bisection Method 2}

Karp's second algorithm partitions the problems by exactly bisecting the area of the rectangle parallel to the shorter side. This produces, however, a more complex situation for the patching algorithm as there is no shared city. 


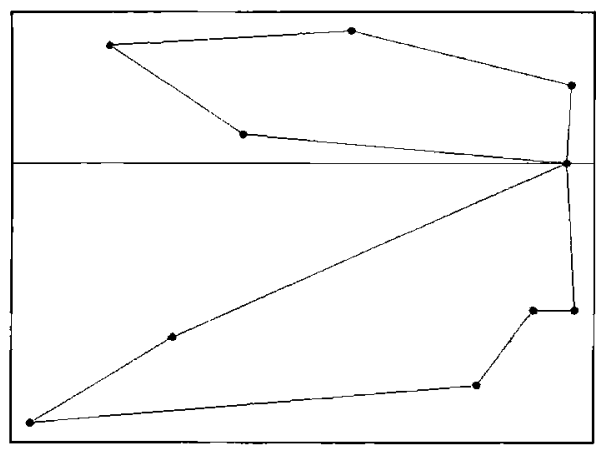

Figure 2. Subproblems solved.

\subsection{Bisection Method 3}

In order to keep the patching algorithm simple, the original bisection method 1 was replaced by the following bisection rule: Rectangles are bisected through the city nearest to the true area bisection line.

In this way a shared city is maintained and, to some degree, the simplest features of the first and second method are combined. The main advantage of this modified bisection method is that the cities in the region of bisection need not be sorted; they are simply partitioned into two sets either side of the bisection line, producing either a left-hand set and a right-hand set, or an upper set and a lower set, depending upon the direction of bisection. The complexity of a single application of this operation is $O(n)$ (instead of $O(n \log n)$ ) and the total cumulative effect is $O(n \log n)$.

\subsection{Solving the Subproblems}

The subproblem size $t$ is kept as small as possible, typically $5 \leq t \leq 8$. We tried various techniques for solving the subproblems, including exhaustive search. However, 2-Opt was chosen as the main method for this preliminary work because of its speed and simplicity and the fact that it can be applied to larger subproblems, without large time penalties, if required.

\subsection{The Simple Patching Algorithm}

Figure 1 shows the bisection technique resulting in a shared city occurring on the line separating each adjacent pair of subproblems. After the subproblems have been solved, as in Figure 2, the four incident edges to the shared city must be reduced to two. This is achieved by the removal of two of the incident edges, one from each subproblem, and the creation of a new edge between the two "stranded" cities. As there are only four possible ways this patching can be done, they are all tried and the one that results in the shortest patched tour is selected. For later purposes the new edge can be added to an edge list $\mathrm{L}$ as a candidate for repair.

Figure 3 illustrates the best patching obtained in this way for the 10 -city problem used in Figure 1 and Figure 2. 


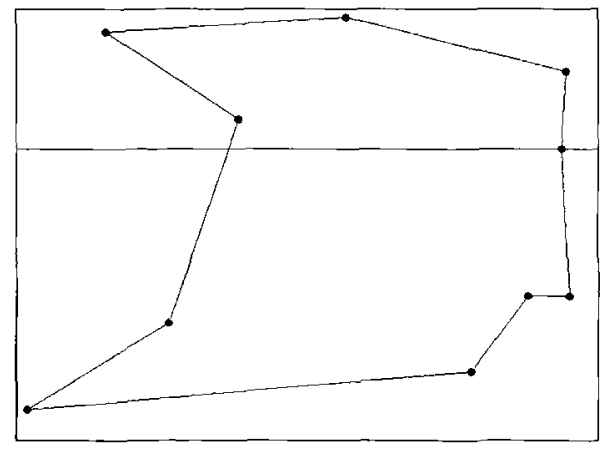

Figure 3. Patched solution.

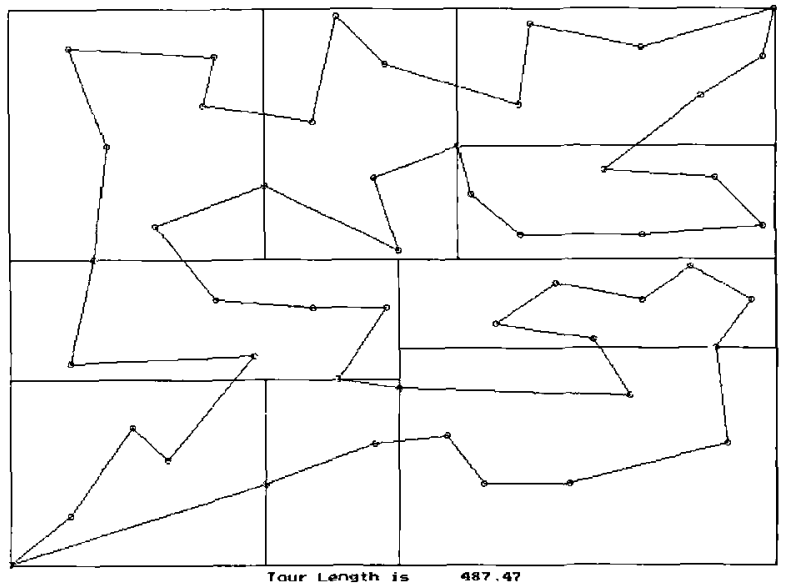

Figure 4. Solution to 50-city problem using Karp's deterministic bisectıon method 1 .

\subsection{Recursive Divide and Conquer}

In Karp's algorithms, the bisection technique is repeated recursively until the individual subproblem sizes are at or below some predetermined maximum value, as illustrated in Figure 4 . When the resulting subtours have been solved, Karp then patches the solutions globally using two operations called Loop and Pass.

The final $E D A C$ algorithm described here differs from Karp's in three important respects:

- A genetic algorithm determines the direction of bisection (horizontal or vertical) used at each stage.

- The patching technique described above is used to join the subproblem solutions recursively in pairs instead of patching globally as Karp does.

- Because simple patching turns out in practice to be a major source of error the new 
edges created by patching (on the list $\mathrm{L}$ ) are reviewed for repair. The repair procedures ultimately used are called Recursive-Fast-2-repair and Far-repair. These will be described later.

\section{Implementation of a Preliminary EDAC Algorithm}

For this study we chose an extremely simple genetic algorithm based on Cavicchio's preselection paradigm (Cavicchio, 1970), in which a child either replaces a weaker parent or dies (in the latter case we still count this evaluation as a trial). Cavicchio's technique in the form used has the virtue of extreme simplicity and low computational overhead while successfully maintaining diversity in the relatively small population of 100 . Gorges-Schleuter, for example, reports excellent results for a closely related algorithm, in which the superior offspring replace one or the other parent (Gorges-Schleuter, 1990).

Our main initial objective is to demonstrate that genetic algorithms have potential in this area; we leave work on improving the genetic algorithm to a later date. The genetic algorithm used for the present is outlined in Algorithm 1. Here there is no need to tune such factors as the crossover rate or the relationship between tour length and fitness.

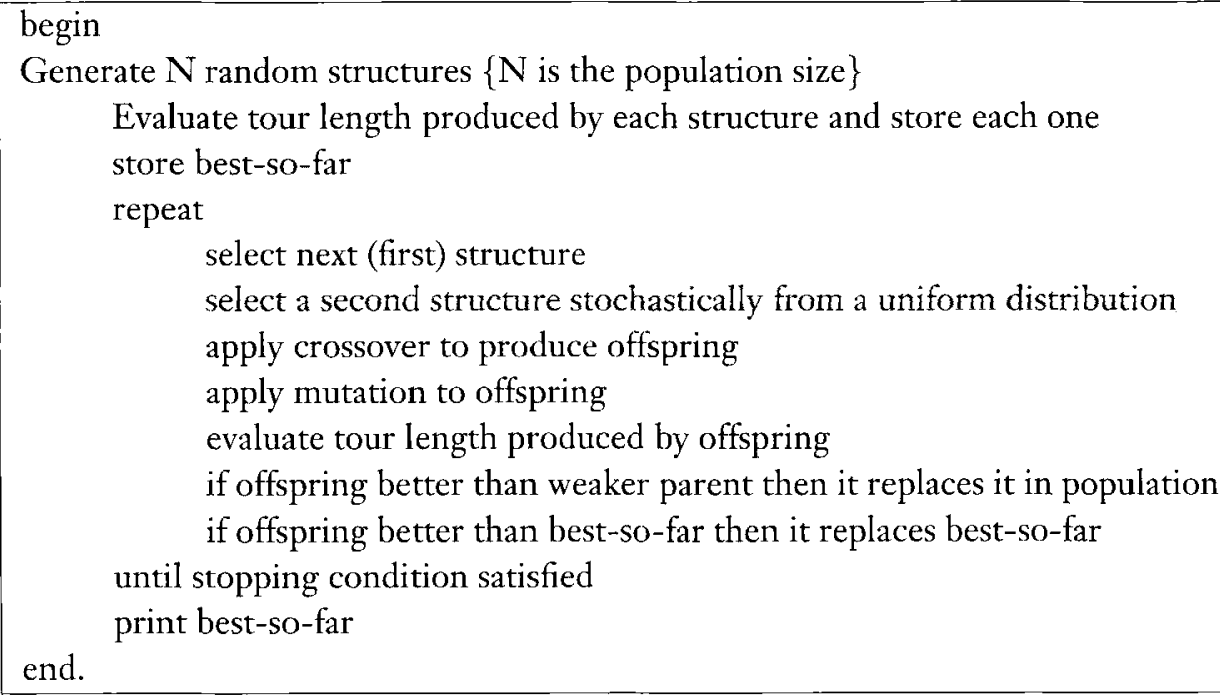

Algorithm 1. The Genetic Algorithm.

\subsection{The Genotype Representation and Crossover}

The data structure for the genotype required some thought. Our initial view favored a binary tree structure in which each node in the tree is labeled with either a "vertical" or "horizontal" cut instruction. This structure lends itself naturally to the recursive nature of both the bisection and the construction of the resulting tour. However, as the tree becomes deeper, the link between a cut instruction at a node and the geometrical region to which that instruction applies becomes progressively more tenuous. Performing a crossover between two binary trees (by exchanging subtrees, for example) could easily produce a child where the subtrees were dissecting completely different geometrical regions for the child than they were for the parents. 


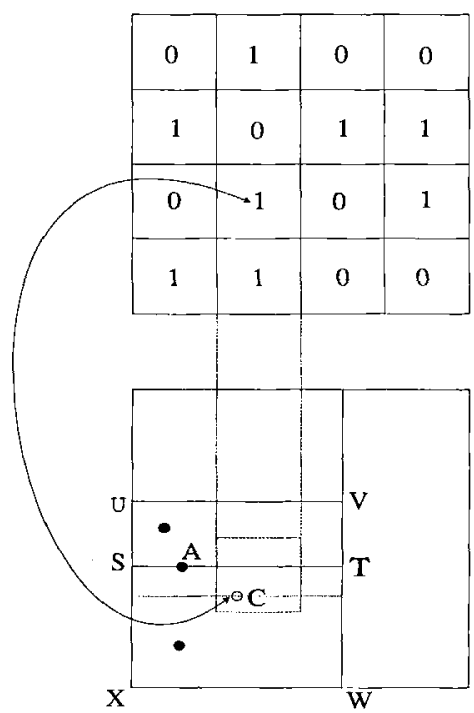

Figure 5. Relationship between the genotype (top) and the direction of bisection ST.

The representation actually used was a $p$ by $p$ binary array that is correlated with the geometrical regions by imagining the array superimposed on the TSP square. Given some rectangle to be bisected, the partitioning algorithm selects the array component that most closely corresponds to the center of the rectangle, and this component (1/0) determines the direction of the current cut (horizontal/vertical). This maintains a close correspondence between the chromosomes of the genotype and the geometrical locality of the center of the rectangle. In the current study we used $40 \leq p \leq 80$.

Figure 5 illustrates the geometrical relationship between the genotype with $p=4$ (top) and the direction of bisection of the rectangle UVWX (bottom). The center of the rectangle UVWX is $\mathrm{C}$, which corresponds to the square indicated in the genotype. The genotype entry of " 1 " denotes a horizontal bisection of the area. The city nearest to the bisector through $\mathrm{C}$ is A, and the horizontal line ST through A is the bisector actually constructed by method 3 (see section 2).

Given that the genotype is a binary array, the crossover becomes relatively trivial, requiring only the swapping of binary elements between two parent arrays. In our current implementation we select the $\mathrm{x}$ or $\mathrm{y}$ axis with equal probability and then choose two cut points at random on the selected axis with the proviso that the distance between the two points must be more than a third and less than two thirds along this axis of the genotype. The reason for the one-third/two-thirds restriction is to ensure that each offspring contains a reasonable proportion of genetic material from each parent, thus attempting to avoid the early proliferation of a few superior individuals.

The first cut relates to the whole region and, as bisection progresses, the region corresponding to a single array element becomes geometrically smaller and the cities within the region less uniformly distributed. Since the genotype is a binary array one can envisage that a suitably modified schema theorem may possibly apply. Although a schema theorem by itself would be no guarantee of progress (Grefenstette \& Baker, 1989), it might be useful 
in the overall scheme of things if the optimal decision to cut horizontally or vertically near any rectangle center is correlated with the distribution of cities. It seems likely that this is the case.

\subsection{The Size of the Genotype}

The array acts as a look-up table for the genetic algorithm with only a few points being accessed for each application of the partition algorithm. In this respect it is analogous to the DNA in natural chromosomes for which only a small part is active in each cell, the remainder being "switched off." Certainly $p$ must be at least $\sqrt{ }(n / t-1)$, but for extreme distributions of cities a given value of $p$ may not provide sufficient resolution and a larger value may be required. Although suitable array sizes for TSP problems of different magnitudes is an obvious area for investigation, it is worth noting that while a population of large arrays would occupy much more memory than a population of small arrays, it would not consume significantly more computing time. More copying would obviously be required to produce the offspring and more mutations to achieve a given mutation rate, but the number of times the genotype is accessed as a look-up table depends only on the number of partitions required for a particular problem and is completely independent of the genotype size.

\subsection{Mutation}

A random point of the array is inverted such that a horizontal instruction becomes a vertical instruction and vice versa. For each genotype created by the genetic algorithm $0.1 \%$ of array components were mutated.

\section{Random Karp-like Solutions versus GA Karp-like Solutions}

If we maintain the subproblem size $t$ and increase the number of cities in the TSP, then a partition better than Karp's becomes progressively harder to find by randomly choosing a horizontal or vertical bisection at each step. If the problem size is $n \sim 2^{k} t$, where $2^{k}$ is the number of subsquares, then the corresponding genotype requires at least $n / t-1$ bits. The size of the partition space is 2 to the power $p^{2}$, which for $p=80$ (the value we used for $n=5000$ ) is approximately $\exp (4436)$. For $n=5000$, the size of permutation search space, roughly estimated using Stirling's formula, is around $\exp (37586)$. Thus, searching partition space is easier than searching permutation space, but still the hard nature of the bisection problem provides sufficient motivation for exploring genetic algorithms as a possible adaptive search technique.

In Figure 6 we present the results of 1000 attempts at dissecting a 500-city problem, "tossing a coin" at each stage to determine whether to perform a horizontal or vertical bisection and then using bisection method 1. Again the subproblem size is about 6 . Not one of the thousand random trials produced a solution as good as the deterministic Karp bisection technique, which gave 127.96 .

A single run of $E D A C$ using the same bisection method, for 100 generations with a population of 100 (10,000 individuals examined), produced a solution of 122.58 ; thus, at $n=500$ the $E D A C$ approach proved capable of improving upon the deterministic Karp algorithm. This was reassuring because it demonstrated that the method had some hope of success. Nevertheless, the solution quality was still unsatisfactory; this led us to search for ways to improve the quality of the Karp-like solutions produced by $E D A C$. 


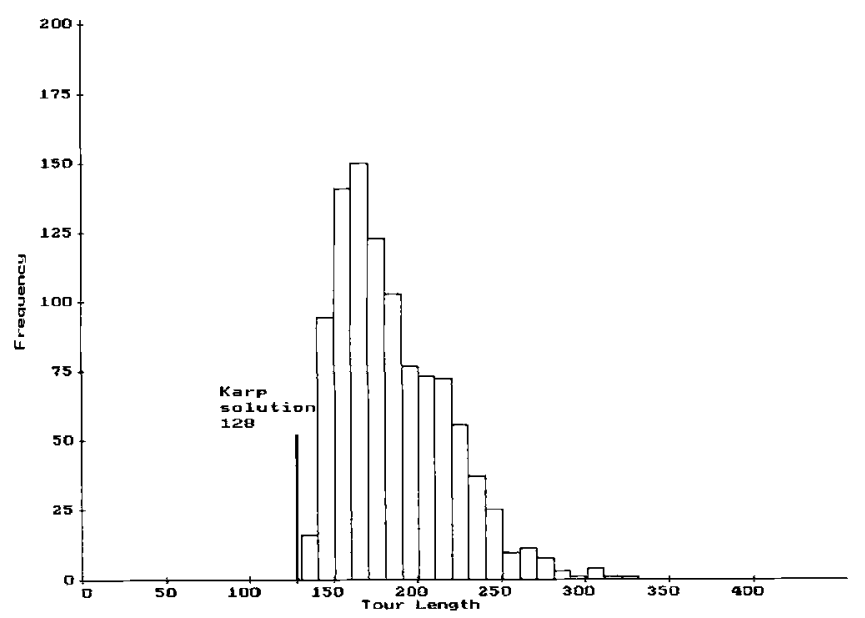

Figure 6. Results of 1000 random dissection experiments on a 500-city problem using simple patching.

\section{Improving the Quality of Karp-like Solutions: Recursive-Fast-2-Repair}

Using bisection method 3 (see section 2) gives an overall improvement in run time without seeming to affect the solution quality much either way, and all subsequent results reported herein used this method. It became clear that, in order to eliminate the more obvious defects introduced by patching, it would be necessary to weaken the link between genotype and phenotype by using a repair mechanism on the Karp-like solutions generated by the genetic algorithm. We have not yet explored all the options in this direction, but in this study we initially opted for a method we called Global-Fast-2-repair, which we subsequently modified to Recursive-Fast-2-repair.

The constraints on the repair technique are fairly obvious: it should address errors typical of Karp-like solutions, it should ideally be $O(n)$, and it should use information that can be acquired at low time cost.

The most basic of the combinatorial tour repair heuristics is $2-O p t$, which proceeds by a series of pairwise edge exchanges called 2-moves. Figure 7 illustrates a 2-move for the intuitive edge-crossing case, but it is possible to effect a 2-move improvement even in cases where the two replaced edges do not cross.

To find a 2-move that decreases the tour length a simple 2-Opt must consider all edge pairs for a possible exchange, which itself requires an $O\left(n^{2}\right)$ calculation. If a 2-move leads to a decrease of the tour length the edge exchange is accepted; this requires inverting and rewriting part of the tour. Once accepted, a single 2-move therefore costs an amount of computation time $d(n)$, which depends on the length of the segment to be inverted (i.e., the quality of the current tour). ${ }^{2}$ If the current tour is very bad, $d(n)$ is proportional to $n$. For good tours, $d(n)$ can be much less, proportional to $n^{\alpha}$, where $\alpha<1$. This leads to an overall time complexity of $O\left(n^{2} d(n)\right.$ ), and it is easy to prove that the worst case analysis is $O\left(n^{3}\right)$ (see Lawler et al., 1985, p. 164).

2 It might appear at first that this cost is implementation-dependent, and may possibly be avoided by skillful use of pointers. However, a number of experiments convinced us that the more tempting alternatives yielded longer run times in practice. 


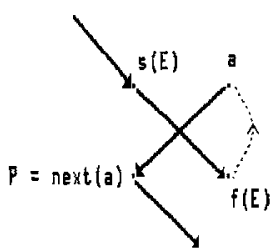

Before

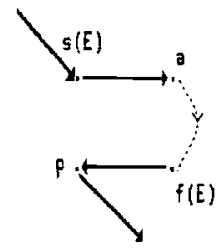

After

Figure 7. A 2-move on edge $\mathrm{E}$ involving a neighbor $a$.

First described by Martin, Otto, and Felten (1992), Fast-2-Opt is a modification of the standard 2-Opt that restricts the number of 2-moves considered. For the geometric TSP, when using 2-Opt it is silly to consider pairs of edges that are far apart in the physical space of the problem. One way in which Fast-2-Opt makes this idea precise is by maintaining a list for each city of the edge lengths to (say) the 10 nearest neighbors, and restricting 2-moves to these edges. Unfortunately, constructing these lists is itself at least an $O\left(n^{2}\right)$ operation if one is not given all the edge lengths to begin with. Fortunately, as shortly described, in the context of an evolutionary search this problem is easily overcome.

To further encourage rapid termination, Martin et al. introduced the guard condition that depends on Min, the minimum edge length of all edges, and $M a x$, the maximum edge length of the current tour. The guard condition is the essence of their Fast-2-Opt since it introduces an element of geometrical locality that restricts the number of cases to be considered. The original guard condition requires that one calculate the minimum possible edge length, an $O\left(n^{2}\right)$ calculation. We replaced this by an estimate based on the initial population, which means we may have missed some 2-move improvements. This estimate could be updated as the evolutionary search progressed.

Since we already have a list $\mathrm{L}$ of potential edges for repair, which is generated by the simple patching process, our first attempt at a repair algorithm consisted of a modification of Fast-2-Opt that, in addition to the nearest neighbor lists, also used the list L. Algorithm 2 contains outline pseudocode for this procedure, which we called Global-Fast-2-repair. A similar routine is also considered by Gorges-Schleuter (1990).

The initial version of Global-Fast-2-repair did not require the $n^{2}$ nearest neighbor calculations. Instead, the nearest neighbors to each city are estimated from the initial population of patched solutions, where each city's neighbors in the tour are candidates for insertion into the nearest neighbor lists found so far (the lists are sorted in increasing order of edge lengths). As the evolutionary search progresses and further neighborhood information becomes available, these lists could be progressively updated. However, comparisons between the initial lists and those generated by the full $O\left(n^{2}\right)$ calculation were quite favorable. Once the initial neighbor lists have been constructed and prior to the start of the genetic algorithm, the initial set of tours was itself subjected to Global-Fast-2-repair, and the tour lengths recorded. Subsequently each new tour generated by the genetic algorithm was subjected to Global-Fast-2-repair using the edge list $\mathrm{L}$ described in section 2.

Plainly, Algorithm 2 terminates (each improvement decreases the tour length and there are only finitely many tour lengths); the important issue is how quickly. The initial length of the active list $\mathrm{L}$ is at most $n / t-1$, where $t$ is the number of cities in each subproblem, but 


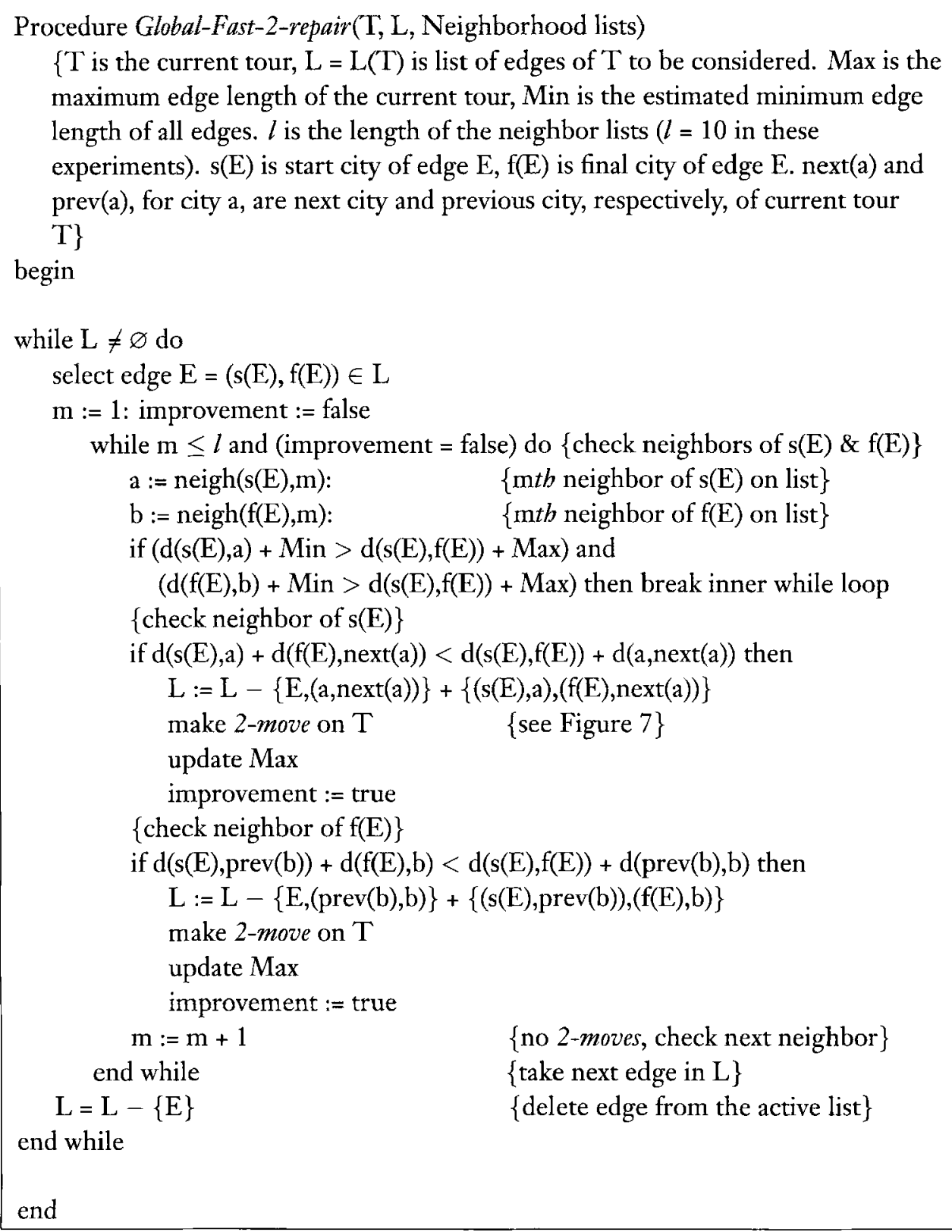

Algorithm 2. Global-Fast 2-repair.

L can sometimes get longer, since the edge (a, next(a)), or (prev(b),b), which is subtracted if present in L, may not (in fact) be in L. If we do not add the second edge, then a much faster, but less accurate procedure, results.

However initial experiments showed that, while Global-Fast-2-repair was successful in lifting the quality of solution from $13 \%$ (using slightly more elaborate patching) to $4-5 \%$ excess, the scaling was poor. Up to $n=5000$ Global-Fast-2-repair was scaling at around 
$O\left(n^{1.7}\right)$, and the exponent seemed to be increasing as $n$ got larger. Not adding the second edge improved the scaling to approximately $O\left(n^{1.3}\right)$, but the solution quality was around $10 \%$ excess. The next step toward improving the situation was to attempt to get as much benefit from 2-moves as possible while limiting the combinatoric growth of cases considered.

We modified Global-Fast-2-repair to become a local procedure, Recursive-Fast-2-repair, which is applied to repairing subsolutions rather than the whole tour. Recursive-Fast-2-repair succeeds each simple patching operation in the recursive construction of the global tour. While the function of Recursive-Fast-2-repair is essentially the same as its global counterpart, its implementation is subtly different. Recursive-Fast-2-repair expends most of its efforts repairing small subproblems, where accepted 2-moves require only short subtour inversions. In addition, each call to Recursive-Fast-2-repair is initiated with an edge list $\mathrm{L}$ containing just one edge, the rogue edge produced by a single simple patching algorithm. Global-Fast-2repair, on the other hand, is characterized by longer subtour inversions and is initiated with an edge list containing all the rogue edges resulting from all the simple patching operations.

Some results obtained from single runs of $E D A C$ with Recursive-Fast-2-repair are presented in Table A-1 (Appendix). A least-squares analysis reveals an empirical scaling of $O\left(n^{1.07}\right)$, and a linear plot results from time versus $n \log n$. Thus, the scaling properties meet our requirements. Unfortunately, the quality of the solutions at $8-10 \%$ excess are considerably worse than those obtained using Global-Fast-2-repair at 4-5\% excess.

\section{Improving the Quality of Karp-like Solutions: Far-Repair}

With a view to further improving the solution quality we developed a low-cost tour improvement heuristic. In essence, the scheme deletes cities from their positions in the current tour and inserts them in new positions whenever this move produces a reduction in the tour length. The algorithm, which we call Far-repair, is applied globally following the construction of the initial tour by simple patching and Recursive-Fast-2-repair.

Algorithm 3 details Far-repair, which obviously has time complexity $O(n)$. Far-repair involves exchanging three edges (a 3-move) and so will repair defects that are beyond the scope of any 2-move.

The lists of nearest neighbors accumulated for the 2-move procedures are employed by Far-repair to ensure that the algorithm does not waste valuable time evaluating potential moves that have little chance of success. Figure 8 shows how a city is tested as a candidate for a Far-move. It is tried first on one side of a near neighbor, then the other. The term "far" repair refers to the fact that individual cities can be moved to new positions in the current tour that are "far away" from their present positions in terms of where they are on the permutation list defining the tour.

\section{Some Preliminary Results}

In practice the simplest 2-Opt has a time complexity of slightly more than $O\left(n^{2}\right)$ (see Figure 9 , bottom trace). Here each data point represents the average for 100 random tours subjected to 2-Opt for fixed problems of size $n=100,200,500,1000,2000$, and 5000, respectively. The line represents the least squares fit and has slope 2.028. Accuracy for the simple 2-Opt is around $8-9 \%$ excess.

Table A-2 (Appendix) gives some results for EDAC with Recursive-Fast-2-repair + Farrepair. These results are plotted in the top trace of Figure 9. The least squares slope is about 1.04 and the accuracy is, at worst, $6 \%$. It may seem counter-intuitive that the scaling 


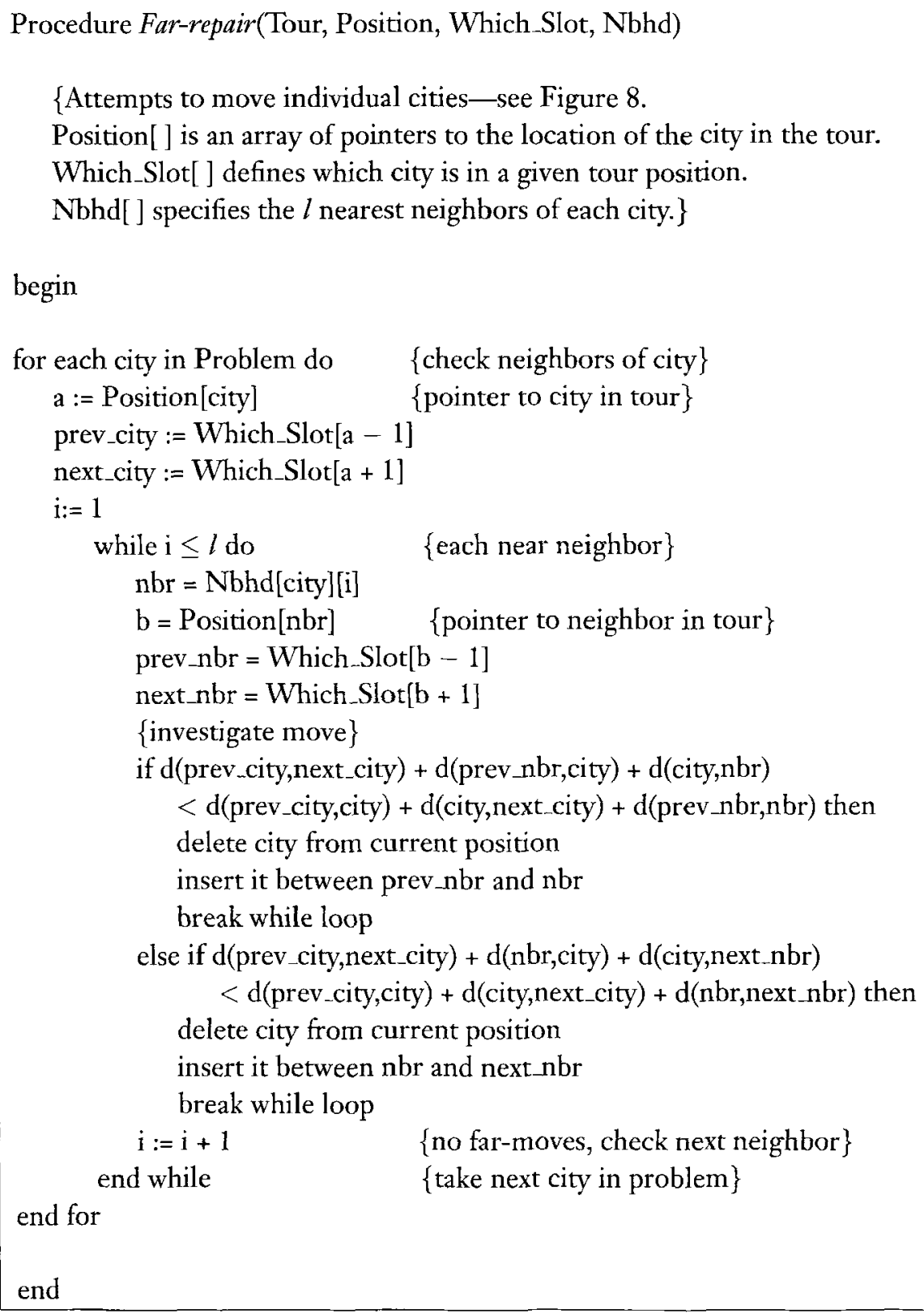

Algorithm 3. Far-repair.

exponent 1.04 (using both repair techniques) is less than 1.07 (when only one is used). Of course, for sufficiently large $n$ the larger exponent must dominate. However, when $n$ is small Far-repair takes up a high proportion of the total CPU time, and as $n$ gets larger this proportion decreases rapidly.

Although these are preliminary results it is quite clear that the general method of approach is viable. Our initial attempt succeeded in the goal of designing a genetic algorithm 

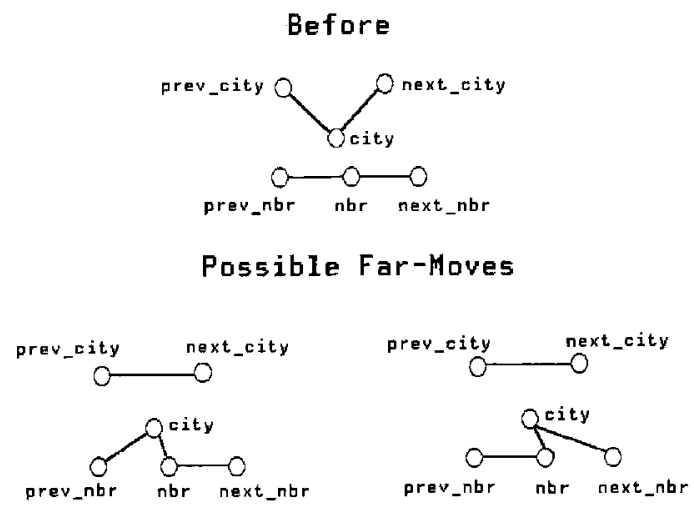

Figure 8. Potential Far-moves.

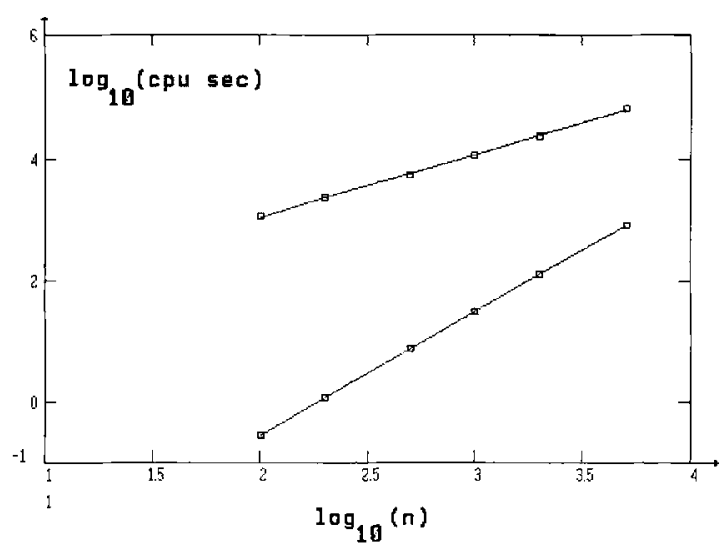

Figure 9. The $E D A C$ (top) and simple 2-Opt (bottom) time complexity (log scales).

capable of reliably giving solutions with around 5-6\% excess for geometric TSP problems involving several thousand cities within 100 generations (10,000 individuals tested).

In Figure 10 the $E D A C$ algorithm has been allowed to run for 200 generations (as opposed to the normal 100) and, although the tour quality is still improving, it is clear that, without more effective repair heuristics, further tour quality improvement will be marginal.

This particular 200-generation run produced a tour having a 5\% excess; see Figure 11. On the downside it is clear that, while viable, the method is probably not yet practical. ${ }^{3}$ The natural comparison would perhaps be with iterated Lin-Kernighan. However, in reviewing the compute-time figures one should bear in mind that we were interested in scaling and made no attempt to optimize the $E D A C$ code.

\footnotetext{
3 For example, wildly extrapolating our figures gives the breakeven point with 2-Opt at around $n=422,800$ requiring some $74 \mathrm{CPU}$ days! Of course, other things would collapse before then.
} 


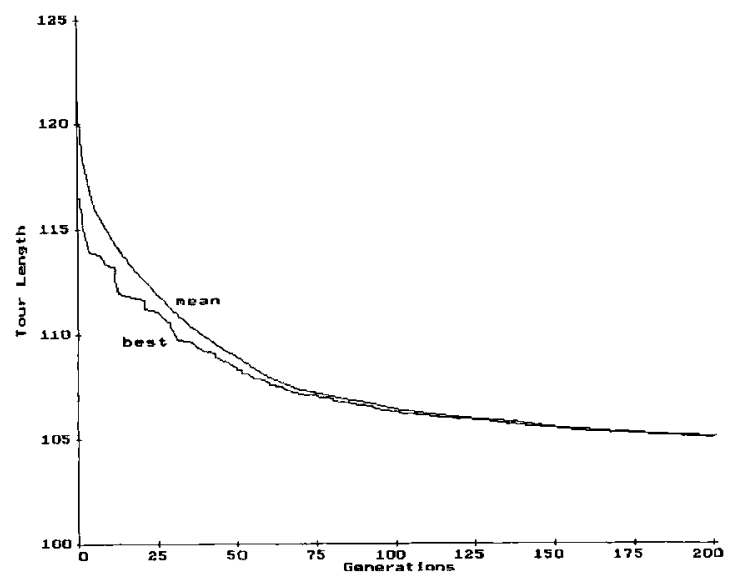

Figure 10. $E D A C$ for 200 generations on a 5000-city problem.

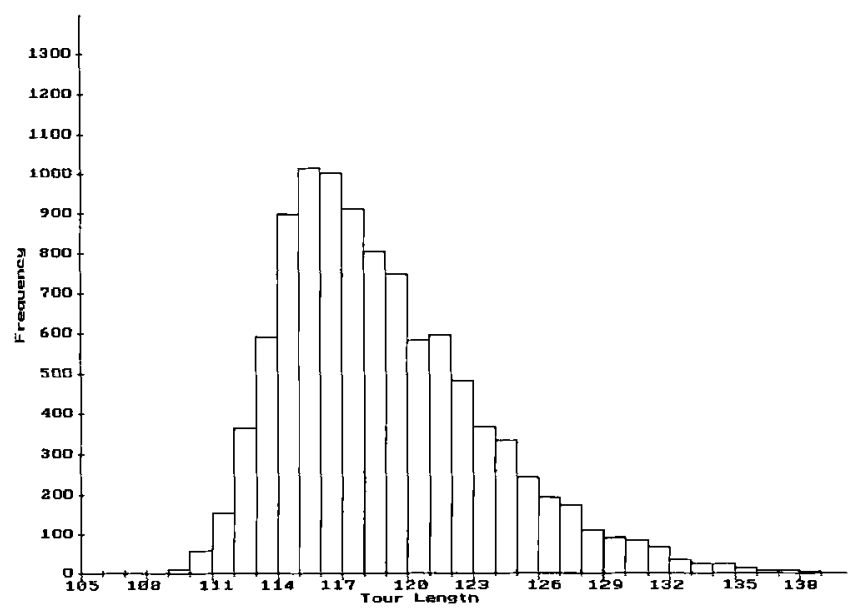

Figure 11. The 200-generation $E D A C 5 \%$ excess solution for a 5000-city problem.

\section{What Is the Overall Contribution of the Genetic Algorithm?}

In order to assess the contribution of the genetic algorithm over and above both random search and Karp's deterministic bisection method, we ran some control experiments. 


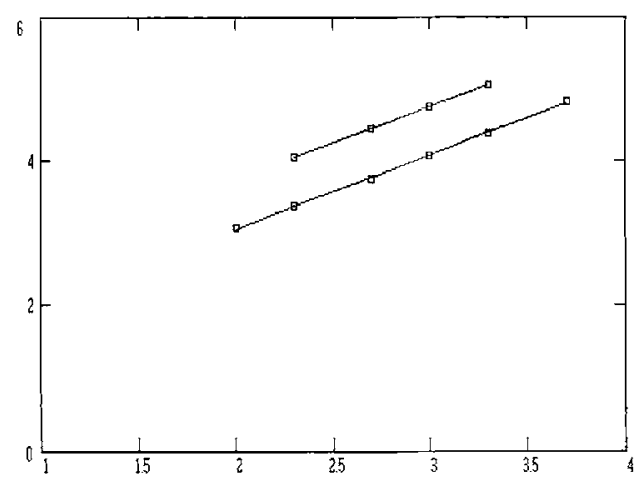

Figure 12. Random search + repair heuristics for a 500-city problem. The deterministic Karp + repair heuristics yield a tour length of 112.33 .

Table 1. Comparing 10,000 random Karp-like solutions + repair beuristics with EDAC + repair beuristics.

\begin{tabular}{||c|c|c|c|c||}
\hline \hline Problem size & Mean & $\begin{array}{c}\text { Standard } \\
\text { Deviation }\end{array}$ & Best & EDAC \\
\hline \hline 500 & 118.87 & 4.66 & 108.91 & 105.34 \\
\hline 1000 & 120.15 & 4.35 & 110.66 & 104.65 \\
\hline 2000 & 120.12 & 4.16 & 112.20 & 104.66 \\
\hline
\end{tabular}

Figure 12 shows a distribution of 10,000 Karp-like random trials on a 500 -city problem. Recursive-Fast-2-repair + Far-repair are used here, and other parameters are set to make the 10,000 trials comparable to a single run of $E D A C$ with these heuristics. (EDAC has a population size of 100 , and runs for 100 generations.)

Table 1 summarizes random search experiments on 1000- and 2000-city problems as well as the 500-city results, and compares these with the results obtained by running $E D A C$ and recording the best solution produced. Entries in the $E D A C$ column represent the mean of 5 runs of $E D A C$ for the 500- and 1000-city problems, and the mean of 2 runs for the 2000-city problem.

Examining the table, for $n=500$ the mean of the distribution of randomly generated Karp-like solutions plus repair heuristics is 118.87 . The best of 10,000 such trials gives a solution 2.13 standard deviations (SD) better than the mean, whereas the same number of evaluations using the $E D A C$ algorithm plus repair heuristics yields an improvement $2.90 \mathrm{SD}$ better than the mean, a difference of 0.77 SD between the two. For 1000 cities this difference is $1.38 \mathrm{SD}$; for 2000 cities it is $1.82 \mathrm{SD}$. The $E D A C$ algorithm is steadily improving its performance relative to random search as the problem size increases. Because the distribution is skewed, the relative improvement using the genetic algorithm is actually better than these figures indicate.

It is interesting to note that the one-shot Karp deterministic algorithm plus repair heuristics yielded a solution of 112.33 on the 500 -city problem. Random search plus repair heuristics did better than this with a best of 108.91 . It would appear that Karp's deterministic 


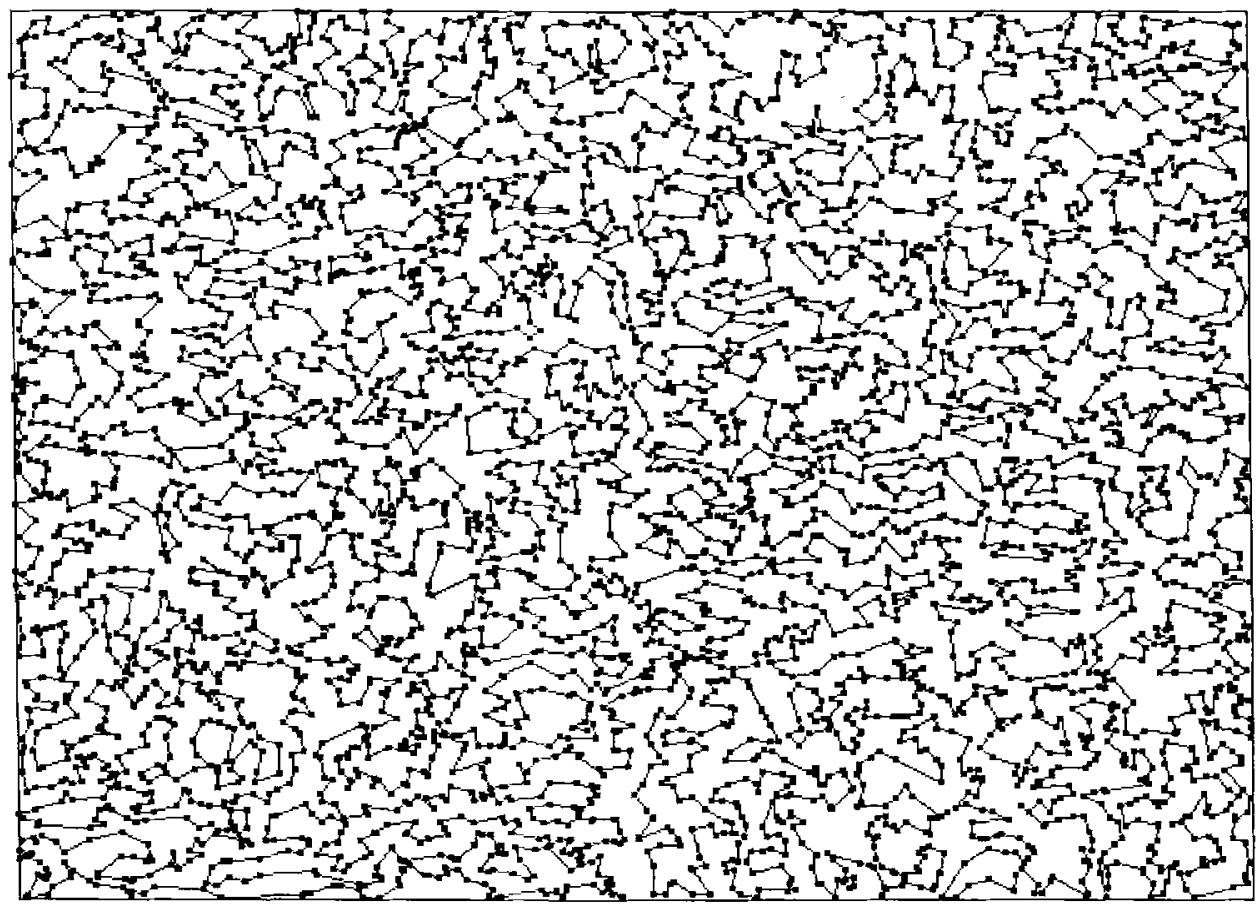

Figure 13. Comparative scaling plots for $E D A C I I$ (top) and the previous results (bottom). The horizontal axis is $\log$ cities, and the vertical axis $\log$ CPU secs.

rule for deciding the direction of bisection becomes less effective as more repair heuristics are added. Fortunately, the same does not seem to be true of the $E D A C$ algorithm.

\section{Conclusions}

Evolutionary divide and conquer offers a new approach for genetic algorithms applied to hard combinatoric search. We have applied this idea to the geometrical TSP and shown it to be viable, if not yet practical. The genotype represents a division of the original problem into subproblems, and the process of constructing a phenotype (tour) from the genotype is analogous to the growth of an individual. To meet the goal of an algorithm with good scaling it is necessary that this growth process scale at $O(n)$ or, at worst, $O\left(n(\log n)^{\alpha}\right)$ for some $\alpha>0$. Since the standard combinatorial local repair heuristics scale at $O\left(n^{2}\right)$ or worse, to satisfy this requirement for an acceptable tour quality we have been obliged to develop geometrically local repair heuristics; one of these heuristics, Far-repair, is presented here. We feel confident that the overall accuracy can be improved by a more sophisticated combination of geometrically local heuristics, and we have a number of promising approaches yet to be explored. In addition we expect that modifications to our genetic algorithm, currently a very simple but nonstandard form, will also yield some improvements.

Once the model is refined, an obvious direction for further work is to parallelize the $E D A C$ algorithm. It is clear that the overall design lends itself to parallelization at several 
levels and in a number of different ways, depending upon the parallel architecture. We plan to explore these possibilities when algorithm refinement is complete.

Subsequent experiments reveal that using repair combinations of Recursive-Fast-2-repair and an Enbanced-Far-repair the EDACII algorithm consistently produces solutions at the $1 \%$ level. For example, for a 2000-city problem we obtained a solution of 100.00 and in other large problems solutions with a negative excess. For this variation of the algorithm the scaling is preserved (the least-squares line has slope 1.014). See Figure 13 and Table A-3 (see Appendix) for preliminary results. However, the overall run times are approximately 5 times higher.

We are thus moving into a phase where we need more accurate estimates of the optimum tour length and have implemented variations of the Held-Karp lower bound. Similarly, it would be extremely useful to have a more accurate estimate of Stein's constant. The difficulty in approaching this empirically is that so few exact solutions are known for very large problems of the right type (uniformly random distributions of cities).

The Enhanced-Far-repair heuristics in version $E D A C I I$ attempt to gain improvements by moving very small groups, as well as individual cities. Despite their obvious success in buying an improved solution quality, we have recently come to consider our recipes of global Far-repair combinations to be the least elegant part of the implementation.

The idea behind Recursive-Fast-2-repair is to exploit the recursive structure of a Karplike tour, and so limit the combinatorial growth of 2-moves when Fast-2-repair is called. This seems to us more in keeping with the divide-and-conquer paradigm. Moreover, this idea is capable of generalization in the sense that it can be applied to any combinatorial repair heuristic. With this in mind, we are now considering more powerful recursive repair mechanisms. We hope the mix-and-match combinations of various types of 2-repair and 3-repair can then be discarded and the resulting algorithm will be more accurate and less time-consuming.

\section{Acknowledgments}

We should like to thank Heinz W. Mühlenbein of GMD for first stimulating our interest in parallel algorithms for very large TSP problems, and Martina Gorges-Schleuter for her continued encouragement and many illuminating discussions. We are also grateful for the helpful comments of the referees.

\section{References}

Beardwood, J., Halton, J. H., \& Hammersley, J. M. (1959). 'The shortest path through many points. Proceedings of the Cambridge Philosophical Society 55:299-327.

Cavicchio, D. J. (1970). Adaptive search using simulated evolution. Doctoral dissertation, University of Michigan, Arin Arbor.

Davis, L. (1985). Job shop scheduling with genetic algorithms. In J. Grefenstette (Ed.), Proceedings of an International Conference on Genetic Algoritbms and Their Applications (pp. 136-140). Hillsdale, NJ: Lawrence Erlbaum Associates.

Goldberg, D. E., \& Lingle, R. (1985). Alleles, loci, and the traveling salesman problem. In J. Crrefenstette (Ed.), Proceedings of an International Conference on Genetic Algoritbms and Their Applications (pp. 154-159). Hillsdale, NJ: Lawrence Erlbaum Associates.

Gorges-Schleuter, M. (1990). Genetic algorithms and population structures, a massively parallel algorithm. Unpublished Ph.D. thesis, Department of Computer Science, University of Dortmund, Germany. 
Christine L. Valenzuela and Antonia J. Jones

Grefenstette, J. J., \& Baker, J. E. (1989). How genetic algorithms work: A critical look at implicit parallelism. In J. D. Schaffer (Ed.), Proceedings of the Third International Conference on Genetic Algoritbms (pp. 20-27). San Mateo, CA: Morgan Kaufmann.

Grötschel, M., \& Holland, O. (1991). Solutions of large-scale symmetric traveling salesman problems. Mathematical Programming; 51, 141-202.

Held, M., \& Karp, R. M. (1970). The travelling salesman problem and minimum spanning trees. Operational Research, 18, 1138-1162.

Held, M., \& Karp, R. M. (1971). The travelling salesman problem and minimum spanning trees: part II. Mathematical Programming, 1, 6-25.

Karp, R. M. (1977). Probabilistic analysis of partitioning algorithm for the Travelling-Salesman Problem in the plane. Matbematics of Operations Research, 2(3), 209-224.

Lawler, E. L., Lenstra, J. K., Rinnooy Kan, A. H. G., \& Shmoys, D. B. (Eds). (1985). The travelling salesman problem--A guided tour of combinatoric optimisation. New York: John Wiley \& Sons.

Lin, S., \& Kernighan, B. W. (1973). An effective heuristic algorithm for the travelling salesman problem. Operational Research, 21, 498-516.

Martin, O., Otto, S. W., \& Felten, E. W. (1992). Large-Step Markov chains for the TSP in cooperating local search heuristics. Operations Research Letters, 11(4), 219-224.

Papadimitriou, C. H., \& Steiglitz, K. (1982). Combinatorial optimisation: Algoritbms and complexity. Englewood Cliffs, NJ: Prentice-Hall.

Smith, D. (1985). Bin packing with adaptive search. In J. Grefenstette (Ed.), Proceedings of an International Conference on Genetic Algoritbms and Their Applications (pp. 202-206). Hillsdale, NJ: Lawrence Erlbaum Associates.

Steele,J.M. (1986). Probabilistic algorithm for the directed Travelling Salesman Problem. Mathematics of Operations Research, 11(2), 343-350.

Stein, D. (1977). Scheduling Dial-a-Ride transportation systems: an asymptotic approach. Doctoral dissertation, Harvard University, Cambridge, MA.

Whitley, D. (1989). Scheduling problems and travelling salesman: The genetic edge recombination operator. In J. D. Schaffer (E.), Proceedings of the Third International Conference on Genetic Algoritbms (pp. 133-140). San Mateo, CA: Morgan Kaufmann.

\section{Appendix}

Table A-1. EDAC with Recursive-Fast-2-repair (Sparc 10)-single runs.

\begin{tabular}{||l|l|l|l|c|c||}
\hline \hline $\mathrm{n}$ & $\log \mathrm{n}$ & $\mathrm{p}$ & time (secs) & $\log$ time & best \\
\hline \hline 100 & 2 & 40 & 706.8 & 2.8493 & 101.07 \\
\hline 200 & 2.3010 & 40 & 1504 & 3.1772 & 109.47 \\
\hline 500 & 2.6990 & 40 & 3718.5 & 3.5704 & 108.39 \\
\hline 1000 & 3 & 60 & 8992.9 & 3.9539 & 108.75 \\
\hline 2000 & 3.3010 & 80 & 16908.4 & 4.2281 & 109.24 \\
\hline 5000 & 3.6990 & 80 & 46825.2 & 4.6705 & 110.35 \\
\hline
\end{tabular}


Table A-2. EDAC with Recursive-Fast-2-repair + Far-repair (Sparc 10)-average of 5 runs.

\begin{tabular}{||l|l|c|l|c|l||}
\hline \hline $\mathbf{n}$ & $\log \mathrm{n}$ & $\mathrm{p}$ & time $(\mathrm{sec})$ & $\log$ time & best \\
\hline \hline 100 & 2 & 40 & 1085.298 & 3.0355 & 99.27 \\
\hline 200 & 2.3010 & 40 & 2205.258 & 3.3435 & 105.41 \\
\hline 500 & 2.6990 & 40 & 5473.458 & 3.7383 & 105.34 \\
\hline 1000 & 3 & 60 & 11679 & 4.0674 & 104.65 \\
\hline $2000^{*}$ & 3.3010 & 80 & 23444.4 & 4.3700 & 104.66 \\
\hline $5000^{*}$ & 3.6990 & 80 & 65012.4 & 4.8129 & 105.90 \\
\hline \hline
\end{tabular}

${ }^{*}$ denotes average of 2 runs.

Table A-3. EDACII with Recursive-Fast-2-repair + Enbanced-Far-repair (Sparc 10)-average of 4 runs.

\begin{tabular}{||l|l|l|l|l|l||}
\hline \hline $\mathrm{n}$ & $\log \mathrm{n}$ & $\mathrm{p}$ & time $(\mathrm{sec})$ & $\log$ time & best \\
\hline \hline 200 & 2.3010 & 40 & 11089.9 & 4.04493 & 101.81 \\
\hline 500 & 2.6990 & 40 & 28013.2 & 4.4474 & 100.71 \\
\hline 1000 & 3 & 80 & 57071.5 & 4.75642 & 99.62 \\
\hline $2000^{*}$ & 3.3010 & 80 & 114127.2 & 5.05739 & 100.00 \\
\hline
\end{tabular}

* single run. 